13,01

\title{
Квантовое трение и трение ван-дер-Ваальса в конфигурациях частица-пластина и пластина-пластина: нелокальные эффекты
}

\author{
(C) Г.В. Дедков, А.А. Кясов \\ Кабардино-Балкарский государственный университет им. Х.М. Бербекова, \\ Нальчик, Россия \\ E-mail: gv_dedkov@mail.ru; aa_kyasov@mail.ru \\ Поступила в Редакцию 14 февраля 2020 г. \\ В окончательной редакции 14 февраля 2020 г. \\ Принята к печати 30 марта 2020 г.
}

\begin{abstract}
В нерелятивистском приближении флуктуационной электродинамики, с использованием модели зеркального отражения и нелокальной диэлектрической проницаемости металла, получены простые аналитические выражения для сил трения в системах частица-пластина и пластина-пластина при относительном движении тел с постоянной скоростью. Показано, что при расстояниях около $1-10 \mathrm{~nm}$ для наночастицы Аи (или золотой пластины), движущейся вблизи другой такой же пластины в состоянии покоя, диссипативные силы на 2-4 порядка выше, чем в случае, когда используется локальная диэлектрическая функция Друде.
\end{abstract}

Ключевые слова: трение Ван-дер-Ваальса, квантовое трение, силы Казимира-Лифшица, нелокальные эффекты.

DOI: $10.21883 /$ FTT.2020.08.49620.032

\section{1. Введение}

Детальное описание сил трения ван-дер-Ваальса (и квантового трения), а также соответствующих диссипативных эффектов в наноструктурах имеет большое фундаментальное и практическое значение в связи с интенсивным развитием микро- и нанотехнологии, поскольку они могут влиять на функционирование микромеханических устройств (МЭМС) [1]. Однако до недавнего времени даже результаты теоретических расчетов этих сил в простейших конфигурациях (частицапластина, пластина-пластина) были предметом интенсивного обсуждения (см. [2-4] и соответствующие ссылки). Практически отсутствуют и надежные экспериментальные измерения сил данного типа, что резко контрастирует с измерениями статических консервативных сил ван-дер-Ваальса и Казимира-Лифшица $[5,6]$, имеющих одинаковое происхождение. Все вышеупомянутые силы зависят от вида диэлектрических проницаемостей взаимодействующих тел. В случае консервативных сил вандер-Ваальса (Казимира-Лифшица), как показано в [6,7], роль пространственной дисперсии (ПД) диэлектрической проницаемости материалов относительно мала, но в случае квантового трения ПД проявляется значительно сильнее $[8,9]$. Это связано с возможностью генерации электрон-дырочных пар при относительном движении тел (даже с небольшой скоростью).

Целью данной работы является расчет силы трения ван-дер-Ваальса (с линейной зависимостью от скорости при конечной температуре) и квантовой силы трения (с кубической зависимостью от скорости при нулевой температуре) в рамках нерелятивистского приближения флуктуационной электродинамики и модели зеркального отражения (МЗО) [10,11]. Хорошо известно, что МЗО позволяет описывать многие свойства реальных поверхностей и успешно используется для расчета потерь энергии ускоренных ионов вблизи поверхности твердых тел и динамики движения электронов, захваченных кильватерным потенциалом движущихся ионов в веществе [12].

На основе решения задачи о взаимодействии малой частицы с пластиной рассматривается задача о взаимодействии двух толстых пластин в относительном движении. При этом используется ,принцип соответствия“ между конфигурациями частица-пластина и пластина-пластина [13]. В отличие от работ $[8,9]$, в которых рассматривались силы квантового трения между двумя пластинами и при взаимодействии атома с поверхностью, использование реалистичного приближения для нелокальной диэлектрической функции металла позволяет проводить аналитические и численные расчеты этой силы, а также (в общем случае) силы трения ван-дер-Ваальса, более простым образом при произвольных температурах взаимодействующих тел. Проводится детальное численное сравнение нелокальных сил трения с результатами локальной теории при взаимодействии наночастиц золота с поверхностью золота и взаимодействии двух пластин золота. Показано, что учет нелокальности в этих случаях приводит к увеличению сил трения на 2-4 порядка величины.

\section{2. Основные теоретические соотношения}

На рис. 1 показана схема МЗО и движения частицы с флуктуационным дипольным моментом $\mathbf{d}(t)$ относитель- 


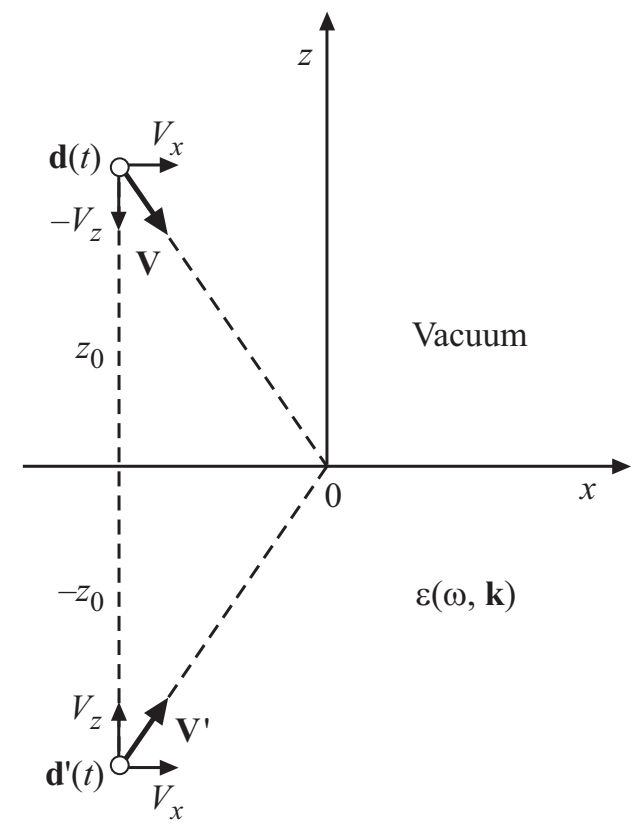

Рис. 1. Схема МЗО и используемая координатная система.

но поверхности поляризующейся пластины. В вакуумной области электрическое поле создается диполем $\mathbf{d}(t)$ c декартовыми компонентами $V_{x}, 0,-V_{z}$ вектора скорости $\mathbf{V}$, идентичным ему диполем с компонентами $\left(V_{x}, 0, V_{z}\right)$ вектора скорости $\mathbf{V}^{\prime}$, зеркально-симметричным относительно плоскости $z=0$, и фиктивной плотностью заряда $\rho_{s}(x, y, t) \delta(z)$ на плоскости $z=0$. Введение фиктивной плотности заряда необходимо для выполнения граничных условий непрерывности электрического потенциала и индукции электрического поля $[10,11]$. Поле внутри пластины $(z<0)$ также создается плотностью заряда $\rho_{s}(x, y, t) \delta(z)$.

Задавая векторы поляризации дипольных источников в виде $\mathbf{P}=\mathbf{d}(t) \delta(\mathbf{r}-\mathbf{V} t)$ и $\mathbf{P}^{\prime}=\mathbf{d}(t) \delta\left(\mathbf{r}-\mathbf{V}^{\prime} t\right)$, и разлагая электрический потенциал $\Phi$ и соответствующие плотности заряда $-\operatorname{div} \mathbf{P},-\operatorname{div} \mathbf{P}^{\prime}, \rho_{s}(x, y, t) \delta(z)$ в интегралы Фурье по частоте $\omega$ и трехмерному волновому вектору $\mathbf{k}=\left(\mathbf{q}, k_{z}\right)=\left(k_{x}, k_{y}, k_{z}\right)$, решение уравнения Пуассона $\Delta \Phi=-4 \pi \rho$ для фурье-компонент потенциала при $z>0$ и $z<0$ запишем в виде

$$
\begin{gathered}
\Phi(\omega, \mathbf{k})=-\frac{4 \pi}{k^{2}}\left[i \mathbf{k} \mathbf{d}(\omega-\mathbf{k} \mathbf{V})+i \mathbf{k d}\left(\omega-\mathbf{k V}^{\prime}\right)\right. \\
\left.+\rho_{s}(\omega, q)\right], \quad z>0 \\
\Phi(\omega, \mathbf{k})=\frac{4 \pi \rho_{s}(\omega, q)}{k^{2} \varepsilon(\omega, \mathbf{k})}, \quad z<0
\end{gathered}
$$

где $\mathbf{d}(\omega-\mathbf{k V}), \mathbf{d}\left(\omega-\mathbf{k V}^{\prime}\right)$ и $\rho_{s}(\omega, q)$ - фурье-компоненты дипольных моментов и $\rho_{s}(x, y, t)$.
Условие непрерывности потенциала $\Phi$ на границе $z=0$ определяет величину $\rho_{s}(\omega, q)$ :

$$
\begin{gathered}
\rho_{s}(\omega, q)=-\frac{q}{\pi+q I_{0}} \int_{-\infty}^{\infty} \frac{d k_{z}}{q^{2}+k_{z}^{2}} \\
\times\left[i \mathbf{k d}\left(\omega-q_{x} V_{x}+k_{z} V_{z}\right)+i \mathbf{k d}\left(\omega-q_{x} V_{x}-k_{z} V_{z}\right)\right] \\
=-\frac{q}{\pi+q I_{0}}\left[i \mathbf{q d}\left(\omega^{+}-q_{x} V_{x}\right)+i \mathbf{q d}\left(\omega^{-}-q_{x} V_{x}\right)\right. \\
\left.+q d_{z}\left(\omega^{+}-q_{x} V_{x}\right)+q d_{z}\left(\omega^{-}-q_{x} V_{x}\right)\right], \\
I_{0}=\int_{0}^{\infty} \frac{d k_{z}}{k^{2} \varepsilon(\omega, \mathbf{k})},
\end{gathered}
$$

где $\omega^{ \pm}=\omega \pm i q V_{z}$.

При вычислении интеграла (3) он рассматривается как предел при $z \rightarrow 0$ от такого же выражения с дополнительным множителем $\exp \left(i k_{z} z\right)$ в подынтегральном выражении, а интегрирование по $k_{z}$ выполняется вдоль контура, включающего вещественную ось и верхнюю полуокружность комплексной плоскости. Кроме того, используется модификация $\mathbf{k d}=\mathbf{q d}-k_{z} d_{z}$ скалярного произведения [14], необходимая для согласования результатов с точным решением для потенциала, полученного при использовании локальной диэлектрической проницаемости. Без этой модификации вклад флуктуационного дипольного момента с компонентой $d_{z}$ зануляется. Граничное условие непрерывности индукции на границе $z=0$ выполняется автоматически, поскольку составляющая потенциала (1) с фурье-компонентой, обусловленной вкладом диполей, симметрична относительно границы $z=0$ и имеет нулевую производную по координате $z$, а вклады в индукцию от фиктивной плотности заряда при $z \rightarrow \pm 0$ взаимно сокращаются. В рамках МЗО любое распределение заряда, связанное с движущейся частицей, обладает такими же свойствами. Фурье-компонента $\Phi^{i n d}(\omega, \mathbf{k})$ потенциала, индуцированного в вакуумной области, определяется путем вычитания из $\Phi(\omega, \mathbf{k})$ фурье-компоненты $\Phi^{v a c}(\omega, \mathbf{k})$ потенциала для частицы, движущейся в вакууме. Для этого нужно сделать замену $\varepsilon(\omega, \mathbf{k}) \rightarrow 1$ в $(1)-(4)$.

В результате получаем

$$
\begin{gathered}
\Phi^{i n d}(\omega, \mathbf{k})=\frac{2 \pi}{k^{2}} \Delta(\omega, q) \cdot\left[i \mathbf{q} \mathbf{d}\left(\omega^{+}-q_{x} V_{x}\right)+i \mathbf{q d}\left(\omega^{-}-q_{x} V_{x}\right)\right. \\
\left.+q d_{z}\left(\omega^{+}-q_{x} V_{x}\right)+q d_{z}\left(\omega^{-}-q_{x} V_{x}\right)\right], \\
\Delta(\omega, q)=\frac{\pi-q I_{0}}{\pi+q I_{0}} .
\end{gathered}
$$

Принимая во внимание (5), выражение для индуцированного потенциала в точке $(\mathbf{r}, t)=(\mathbf{R}, z, t)=(x, y, z, t)$ вакуумной области принимает вид

$$
\begin{aligned}
\Phi^{i n d}(\mathbf{R}, z, t)= & \frac{1}{(2 \pi)^{4}} \int d \omega d^{2} q d k_{z} \\
& \times \Phi^{i n d}(\omega, \mathbf{k}) \exp \left(i\left(\mathbf{q} \mathbf{R}+k_{z} z-\omega t\right)\right) .
\end{aligned}
$$


Если частица движется параллельно поверхности, то, подставляя (5) в (7), необходимо перейти к пределу $V_{z} \rightarrow 0, \quad V_{x} \rightarrow V$ при условии $V_{z} t \rightarrow \pm z_{0}$, где $z_{0}-$ расстояние частицы от поверхности. После этого, интегрируя (7) по $k_{z}$ так же, как и в (3), получаем фурьекомпоненту потенциала с разложением по частоте и двумерному волновому вектору:

$$
\begin{aligned}
& \Phi^{i n d}(\omega, q, z)=\frac{2 \pi}{q} \Delta(\omega, q) e^{-q\left(z+z_{0}\right)}\left[i q_{x} d_{x}\left(\omega-q_{x} V\right)\right. \\
& \left.+i q_{y} d_{y}\left(\omega-q_{x} V\right)+q d_{z}\left(\omega-q_{x} V\right)\right] .
\end{aligned}
$$

Формула (8) совпадает с точным решением электродинамической задачи в случае параллельного движения, когда используется локальная диэлектрическая проницаемость $\varepsilon(\omega)$ [14]. Используя (8), дальнейший расчет диссипативной силы ван-дер-Ваальса, действующей на частицу, полностью повторяет расчет с локальной функцией $\varepsilon(\omega)[3]$. Выражая диэлектрический отклик частицы через частотно-зависящую поляризуемость $\alpha(\omega)$, получаем следующий результат для силы $F_{x}\left(T_{1}\right.$ и $T_{2}-$ температуры частицы и пластины в энергетических единицах, $\left.\omega_{+}=\omega+q_{x} V\right)$

$$
\begin{aligned}
F_{x}= & -\frac{\hbar}{\pi^{2}} \int_{0}^{\infty} \int_{-\infty}^{+\infty} d q_{x} q_{x} \int_{-\infty}^{+\infty} d q_{y} q e^{-2 q z_{0}} \operatorname{Im} \Delta(\omega, q) \operatorname{Im} \alpha\left(\omega_{+}\right) \\
& \times\left[\operatorname{coth}\left(\hbar \omega / 2 T_{2}\right)-\operatorname{coth}\left(\hbar \omega_{+} / 2 T_{1}\right)\right]
\end{aligned}
$$

В линейном приближении по скорости (при $T_{1}=T_{2}$ $=T)$ из (9) следует

$$
\begin{aligned}
F_{x}= & -\frac{1}{2 \pi}\left(\frac{\hbar^{2} V}{T}\right) \int_{0}^{\infty} d \omega \int_{0}^{\infty} d q q^{4} e^{-2 q z_{0}} \\
& \times \operatorname{Im} \Delta(\omega, q) \operatorname{Im} \alpha(\omega) \sinh ^{-2}(\hbar \omega / 2 T),
\end{aligned}
$$

а в пределе квантового трения $(T=0)$, соответственно,

$$
\begin{aligned}
F_{x}= & \frac{4 \hbar}{\pi^{2}} \int_{0}^{\infty} d q_{x} q_{x} \int_{0}^{\infty} d q_{y} q e^{-2 q z_{0}} \\
& \times \int_{0}^{q_{x} V} d \omega \operatorname{Im} \alpha\left(\omega-q_{x} V\right) \operatorname{Im} \Delta(\omega, q) .
\end{aligned}
$$

Формулы (9)-(11), естественно, полностью совпадают с результатами других авторов, полученными для локальной диэлектрической проницаемости материала пластины (см. [2-4] и ссылки), когда $\Delta(\omega, q) \rightarrow(\varepsilon(\omega)-1) /(\varepsilon(\omega)+1)$

\section{3. Сферическая частица над металлической поверхностью}

Для практического расчета силы $F_{x}$ по формулам (10), (11) используем выражение для поляризуе- мости $\alpha(\omega)=R^{3}(\varepsilon(\omega)-1) /(\varepsilon(\omega)+2)$ сферической частицы с радиусом $R$ и диэлектрической проницаемостью Друде $\varepsilon(\omega)=1-\omega_{p}^{2} / \omega(\omega+i \gamma)$, где $\omega_{p}$ и $\gamma-$ частота плазмы и коэффициент затухания $\left(\omega_{p}=9 \mathrm{eV}\right.$ и $\gamma=30 \mathrm{meV}$ для золота). Для функции $\varepsilon(\omega, \mathbf{k})$ металлической пластины используем известное приближение [15], учитывающее низкочастотную асимптотику диэлектрической проницаемости Линдхарда, генерацию электрон-дырочных пар и плазмонов

$$
\varepsilon(\omega, \mathbf{k})=1+\frac{\omega_{p}^{2}}{\left\{s^{2} k^{2}\left[1-i \pi \omega \theta\left(2 k_{F}-k\right) / 2 k V_{F}\right]-\omega(\omega+i \gamma)\right\}},
$$

где $k_{F}-$ волновой вектор Ферми, $s=V_{F} / 3, V_{F}-$ скорость Ферми, $\theta(x)$ - единичная функция Хевисайда. Формула (12) отличается от известного гидродинамического приближения для диэлектрической функции наличием в знаменателе члена с тэта-функцией. Она хорошо зарекомендовала себя в расчетах тормозной способности ионов низких энергий при скользящем движении вблизи поверхности [11].

Низкочастотное разложение интеграла (4) приводит к выражению $I_{0}=A+B+C$, в котором

$$
\begin{gathered}
A=\pi k_{T F}^{-1}\left(1+x^{2}\right)^{-3 / 2} \\
B=-i\left(\frac{\pi}{k_{T F}}\right)\left(\frac{\omega}{\omega_{p}}\right) \\
\times\left[\frac{\left(2+x^{2}\right)}{2\left(1+x^{2}\right)^{3 / 2}} \ln \left(\frac{p \sqrt{1+x^{2}}+\sqrt{p^{2}-x^{2}}}{p \sqrt{1+x^{2}}-\sqrt{p^{2}-x^{2}}}\right)\right. \\
\left.-\frac{p \sqrt{p^{2}-x^{2}}}{\left(1+p^{2}\right) \sqrt{1+x^{2}}}\right] \theta(p-x) \\
C=-i\left(\frac{\pi}{k_{T F}}\right)\left(\frac{\gamma \omega}{\omega_{p}^{2}}\right)\left[\frac{\sqrt{1+x^{2}}-x}{x \sqrt{1+x^{2}}+x}-\frac{1}{2\left(1+x^{2}\right)^{3 / 2}}\right] .
\end{gathered}
$$

Здесь $\quad x=q / k_{T F}, \quad k_{T F}=\sqrt{3} \omega_{p} / V_{F}-\quad$ обратная длина экранирования Томаса-Ферми, $\quad p=2 k_{F} / k_{T F}$ $=\sqrt[3]{3 \pi^{2} / 2} \sqrt{a_{B} / r_{s}}, a_{B}$ и $r_{s}-$ боровский радиус и параметр модели „желе“ $\left(r_{s} / a_{B}=3.01\right.$ и $p=1.415$ для золота). Мы также используем одинаковое значение фактора затухания $\gamma$ в выражении для $\varepsilon(\omega)$ и в (12). Следует отметить, что в формуле (14) выражение в квадратных скобках несколько отличается от аналогичного, приведенного в работе [11]. Кроме того, в [11] отсутствует фактор $\theta(p-x)$. Учитывая (6), (13)-(15), получим следующее выражение для функции диэлектрического отклика поверхности, $\Delta(\omega, q)$ :

$$
\Delta(\omega, q)=\frac{1-\left(\omega / \omega_{p}\right)^{2} S^{2}(x)+2 i\left(\omega / \omega_{p}\right) S(x) \sqrt{1+x^{2}}}{\left(\sqrt{1+x^{2}}+x\right)^{2}+\left(\omega / \omega_{p}\right)^{2} S^{2}(x)}
$$




$$
\begin{aligned}
S(x)= & {\left[\frac{x\left(2+x^{2}\right)}{2\left(1+x^{2}\right)} \ln \left(\frac{p \sqrt{1+x^{2}}+\sqrt{p^{2}-x^{2}}}{p \sqrt{1+x^{2}}-\sqrt{p^{2}-x^{2}}}\right)\right.} \\
& \left.-\frac{x p \sqrt{p^{2}-x^{2}}}{\left(1+p^{2}\right) \sqrt{1+x^{2}}}\right] \theta(p-x) \\
& +\beta\left[\sqrt{1+x^{2}}-x-\frac{2}{2\left(1+x^{2}\right)}\right] .
\end{aligned}
$$

При записи $S(x)$ в (17) и далее, для простоты, не указывается аргумент $\beta=\gamma / \omega_{p}$. В случае малых скоростей частиц $V \ll V_{F}$ и $S(x) \sim 1 \quad\left(V_{F}-\right.$ скорость Ферми), члены, пропорциональные $\omega^{2}$ в (14), можно не учитывать, поскольку в интегралы (10), (11) дают вклад только низкие частоты $\omega \ll \omega_{p}$. Аналогично, для $\operatorname{Im} \alpha$ можно использовать более простое выражение $\operatorname{Im} \alpha \approx 3 i \gamma \omega / \omega_{p}^{2}$. Тогда, в результате интегрирования по частоте $\omega$, формулы (10), (11) принимают вид

$$
\begin{aligned}
& F_{x}^{(n l)}=-\frac{\pi}{8}\left(\frac{R}{z_{0}}\right)^{3} \frac{\hbar \gamma}{z_{0}^{2}}\left(\frac{T}{\hbar \omega_{p}}\right)^{2} \frac{V}{\omega_{p}} f_{1}\left(2 k_{T F} z_{0}\right), \\
& F_{x}^{(n l)}=-\frac{1}{256 \pi}\left(\frac{R}{z_{0}}\right)^{3} \frac{\hbar \gamma}{z_{0}}\left(\frac{V}{\omega_{p} z_{0}}\right)^{3} f_{2}\left(2 k_{T F} z_{0}\right),
\end{aligned}
$$

где функции $f_{i}(x), i=1,2$ определены выражениями

$$
\begin{gathered}
f_{1}(x)=\int_{0}^{\infty} d z z^{4} e^{-z} S_{1}(z / x), \\
f_{2}(x)=\int_{0}^{\infty} d z z^{6} e^{-z} S_{1}(z / x), \\
S_{1}(x)=S(x) \frac{\sqrt{1+x^{2}}}{\left(\sqrt{1+x^{2}}+x\right)^{2}} .
\end{gathered}
$$

Если при вычислении $F_{x}$ используется локальная функция Друде, то $\Delta(\omega, q) \rightarrow(\varepsilon(\omega)-1) /(\varepsilon(\omega)+1)$ и после интегрирования в $(10),(11)$ получим

$$
\begin{gathered}
F_{x}^{(l)}=-3 \pi\left(\frac{R}{z_{0}}\right)^{3} \frac{\hbar \gamma}{z_{0}^{2}}\left(\frac{T}{\hbar \omega_{p}}\right)^{2} \frac{V}{\omega_{p}} \frac{\gamma}{\omega_{p}}, \\
F_{x}^{(l)}=-\frac{45}{16 \pi}\left(\frac{R}{z_{0}}\right)^{3}\left(\frac{V}{\omega_{p} z_{0}}\right)^{3} \frac{\hbar \gamma}{z_{0}} \frac{\gamma}{\omega_{p}} .
\end{gathered}
$$

На рис. 2 показаны зависимости $F_{x}^{(n l)} / F_{x}^{(l)}$, вычисленные в нелокальном и локальном приближении, от параметра $2 k_{T F} z_{0}$. Сплошная и штриховая кривые соответствуют случаю конечной температуры и $T=0$. Заметим, что для золота $2 k_{T F} z_{0}=34$ при $z_{0}=1 \mathrm{~nm}$. Как следует из рис. 2, эффект ПД приводит к возрастанию сил трения более чем на два порядка величины при $z_{0}=1 \div 10 \mathrm{~nm}$. При сравнении отношений сил $F_{x}^{(n l)} / F_{x}^{(l)}$, соответствующих нелокальному и локальному приближениям, вычисленных по формулам (18), (23) и (19), (24), можно видеть, что возрастание силы трения вследствие ПД связано с большой величиной параметра $1 / \beta=\omega_{p} / \gamma \gg 1$. Это согласуется с результатами [9].

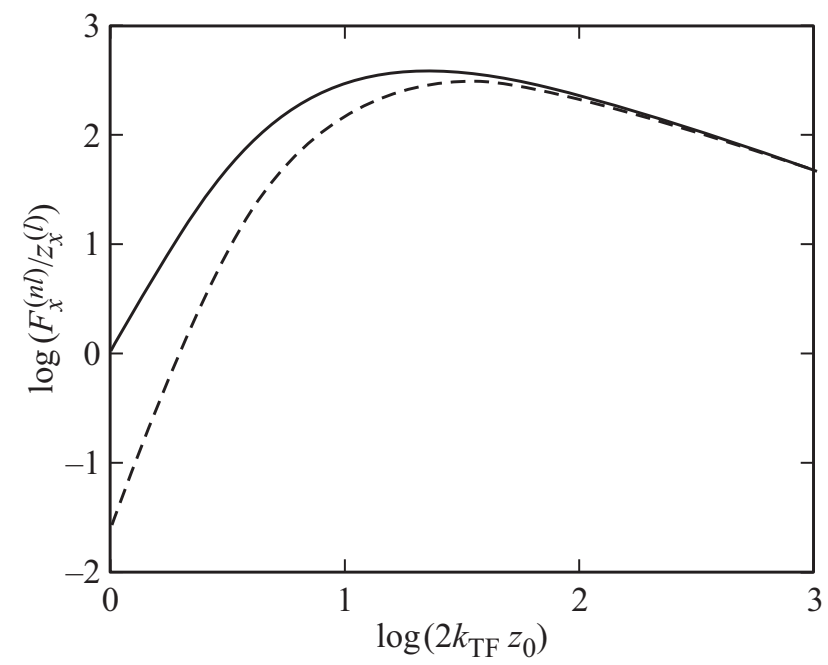

Рис. 2. Зависимость $F_{x}^{(n l)} / F_{x}^{(l)}$ от приведенного расстояния $2 k_{T F} z_{0}$ для наночастицы $\mathrm{Au}$ вблизи поверхности золота. Штриховая линия: сила квантового трения; сплошная линия: сила трения при конечной температуре; $(n l)-$ нелокальное приближение (18), (19); (1) - локальное приближение (23), (24). Логарифмические функции здесь и далее берутся с основанием 10. Для золота, $2 k_{T F} z_{0}=34$ при $z_{0}=1 \mathrm{~nm}$.

\section{4. Две пластины в относительном движении}

Как впервые показал Лифшиц [16], в статическом случае между конфигурациями „пластина-пластина“ (1) и „частица-пластина“ (2) имеется правило соответствия. Оно позволяет получить выражение для силы Казимира-Полдера в конфигурации 2, исходя из соответствующего выражения для силы взаимодействия пластин (полупространств) в конфигурации 1. В соответствии с этим правилом,

$$
\begin{gathered}
\Delta(\omega) \rightarrow 2 \pi n \alpha(\omega), \\
F^{(2)}(z)=-\frac{1}{n S} \frac{d}{d l} F^{(1)}(l)_{l=z},
\end{gathered}
$$

где $\alpha(\omega)$ поляризуемость частицы вещества разреженной среды с плотностью числа частиц $n$, моделирующей материал одной из пластин, а $S$ - площадь поверхности пластин в области вакуумного контакта.

В неравновесных системах, не находящихся в тепловом и (или) динамическом равновесии, соответствующее правило соответствия было обосновано в незапаздывающем пределе $[3,13]$. Было показано, что соотношения (25), (26) выполняются и для других величин, описывающих флуктуационно-электромагнитное взаимодействие, таких как скорость радиационного теплообмена и сила трения. Такое же правило соответствия, очевидно, должно выполняться и в нелокальном случае для существования предельного перехода от нелокального к локальному случаю. Кроме того, соотношения (25), (26) 
могут применяться „в обе стороны“: от конфигурации 1 к конфигурации 2 и наоборот.

Имея это в виду, для учета ПД в конфигурации 1 следует сделать замену $\Delta(\omega) \rightarrow \Delta(\omega, q)$ в формуле (9). Следует отметить, что данный подход принципиально отличается от подхода авторов [4,6] при вычислении сил Казимира-Лифшица (сил притяжения и трения пластин) с учетом ПД, когда нелокальная диэлектрическая функция $\varepsilon(\omega, \mathbf{k})$ (в форме Линдхарда-Мермина [17]) подставлялась непосредственно в выражения для френелевских амплитуд отражения электромагнитных волн в формулах для соответствующих сил, содержащих диэлектрическую функцию.

В нашем случае сила трения, действующая на движущуюся пластину (величины, относящиеся к ней, отмечаются индексом „1“), отнесенная к единице площади контакта, будет совпадать с аналогичным выражением в [3] при условии $\Delta_{1}\left(\omega_{+}\right) \rightarrow \Delta_{1}\left(\omega_{+}, q\right)$ и $\omega_{+}=\omega+q_{x} V$ :

$$
\begin{aligned}
& F_{x}(l)=-\frac{\hbar}{4 \pi^{3}} \int_{0}^{\infty} d \omega \int d^{2} q q_{x} e^{-2 q l} \operatorname{Im} \Delta_{1}\left(\omega_{+}, q\right) \\
& \times \operatorname{Im} \Delta_{2}(\omega, q)|D|^{-2}\left[\operatorname{coth}\left(\hbar \omega / 2 T_{2}\right)-\operatorname{coth}\left(\hbar \omega_{+} / 2 T_{1}\right)\right]
\end{aligned}
$$

где $D=1-\Delta_{1}\left(\omega_{+}, q\right) \Delta_{2}(\omega, q) \exp (-2 q l)$, a $l$ - расстояние между пластинами. Используя (27), выражения для силы трения в линейном приближении по скорости (при $\left.T_{1}=T_{2}=T\right)$ и в случае квантового трения $(T=0)$ запишем в виде $n(\omega)=1 /(\exp (\hbar \omega / T)-1)-$ планковский фактор)

$$
\begin{gathered}
F_{x}=\frac{\hbar V}{2 \pi^{3}} \int_{0}^{\infty} d \omega \frac{d n(\omega)}{d \omega} \int_{0}^{\infty} d^{2} q q_{x} e^{-2 q l} \\
\times \operatorname{Im} \Delta_{1}(\omega, q) \operatorname{Im} \Delta_{2}(\omega, q)|D|^{-2}, \\
F_{x}=\frac{\hbar}{\pi^{3}} \int_{0}^{+\infty} d q_{y} \int_{0}^{\infty} d q_{x} q_{x} e^{-2 q l} \\
\times \int_{0}^{q_{x} V} d \omega \operatorname{Im} \Delta_{1}\left(\omega-q_{x} V, q\right) \operatorname{Im} \Delta_{2}(\omega, q)|D|^{-2} .
\end{gathered}
$$

Следует отметить, что, в отличие от (27) и (29), $D$-фактор в (28) имеет вид $D=1-\Delta_{1}(\omega, q) \Delta_{2}(\omega, q)$ $\times \exp (-2 q l)$. Формулы (28), (29) согласуются с результатами, полученными другими авторами $[2-4,14,18]$ при использовании локальной диэлектрической проницаемости $\varepsilon(\omega)$, а (29) совпадает с формулой (24) в [8], полученной в рамках нелокальной квантово-полевой теории.

Как и в разделе 2 , в случае малых скоростей $\left(V \ll V_{F}\right)$ можно опустить члены, пропорциональные $\omega^{2}$ в (16). Тогда $\Delta_{1,2}(\omega, q) \cong 1, \quad \operatorname{Im} \Delta_{1,2}(\omega, q) \cong 2\left(\omega / \omega_{g}\right) S_{1}(x)$, и при подстановке этих выражений в (28), (29) получим (считая, что обе пластины изготовлены из одинакового материала)

$$
\text { 1) } \begin{aligned}
T_{1}=T_{2} & =T \\
F_{x}^{(n l)} & =-\frac{1}{24}\left(\frac{T}{\hbar \omega_{p}}\right)^{2}\left(\frac{\hbar V}{l^{4}}\right) f_{3}\left(2 k_{T F} l\right) .
\end{aligned}
$$

2) $T_{1}=T_{2}=0$

$$
\begin{gathered}
F_{x}^{(n l)}=-\frac{1}{2^{11} \cdot 3}\left(\frac{V}{\omega_{p} l}\right)^{3}\left(\frac{\hbar \omega_{p}}{l^{3}}\right) f_{4}\left(2 k_{T F} l\right) . \\
f_{3}(x)=\int_{0}^{\infty} d y y^{3} e^{-y} \frac{S_{1}^{2}(y / x)\left(\sqrt{1+(y / x)^{2}}+(y / x)\right)^{4}}{\left[\left(\sqrt{1+(y / x)^{2}}+(y / x)\right)^{4}-e^{-y}\right]^{2}} . \\
f_{4}(x)=\int_{0}^{\infty} d y y^{5} e^{-y} \frac{S_{1}^{2}(y / x)\left(\sqrt{1+(y / x)^{2}}+(y / x)\right)^{4}}{\left[\left(\sqrt{1+(y / x)^{2}}+(y / x)\right)^{4}-e^{-y}\right]^{2}} .
\end{gathered}
$$

В то же время, используя диэлектрическую проницаемость Друде $\varepsilon(\omega)=1-\omega_{p}^{2} / \omega(\omega+i \gamma)$, будем иметь $\Delta_{1,2}(\omega) \cong 1, \operatorname{Im} \Delta_{1,2}(\omega) \cong 2\left(\omega \gamma / \omega_{p}^{2}\right)$ и тогда из $(28),(29)$ следует

$$
\begin{gathered}
F_{x}^{(l)}=-\frac{\xi(3)}{4}\left(\frac{T}{\hbar \omega_{p}}\right)^{2}\left(\frac{\hbar V}{l^{4}}\right)\left(\frac{\gamma}{\omega_{p}}\right)^{2}, \\
F_{x}^{(l)}=-\frac{5 \xi(5)}{2^{8}}\left(\frac{V}{\omega_{p} l}\right)^{3}\left(\frac{\hbar \omega_{p}}{l^{3}}\right)\left(\frac{\gamma}{\omega_{p}}\right)^{2} .
\end{gathered}
$$

где $\xi(3)=1.202$ и $\xi(5)=1.037$ - дзета-функции Римана.

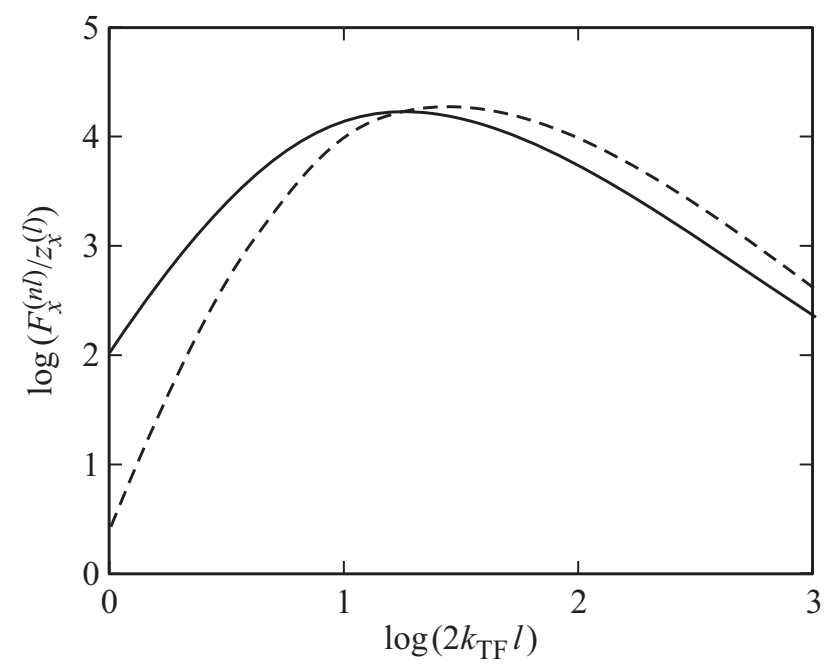

Рис. 3. Зависимость $F_{x}^{n l} / F_{x}^{l}$ как функция приведенного расстояния $2 k_{T F} l$ для двух пластин золота при их относительном движении. Штриховая линия: сила квантового трения; сплошная линия: сила трения при конечной температуре. $(n l)$ - нелокальное приближение $(30),(31),(l)$ - локальное приближение (34), (35). 
На рис. 3 показаны результаты расчета отношения $F_{x}^{(n l)} / F_{x}^{(l)}$ в зависимости от $2 k_{T F} l$ для двух пластин золота при относительном движении с постоянной скоростью. Видно, что в диапазоне $l=1-10 \mathrm{~nm}$ силы трения увеличиваются на четыре порядка, если учитывать ПД. Эффект обусловлен большой величиной фактора $\omega_{p}^{2} / \gamma^{2}$.

\section{5. Обсуждение результатов}

Целесообразно выполнить более детальное сравнение численных значений сил $F_{x}$, найденных с использованием разных подходов. На рис. 4 показана линейная по скорости сила трения между двумя пластинами золота как функция расстояния $l$ при $T_{1}=T_{2}=300 \mathrm{~K}$, $V=1 \mathrm{~m} / \mathrm{s}$. Сплошная толстая кривая показывает нелокальное приближение (формула (30)). Пунктирная толстая кривая и кривая, показанная точками (S-local Drude и $P$-local Drude), показывают результаты расчета с локальной диэлектрической проницаемостью Друде. При этом использовалась модификация общей формулы для силы трения теории Рытова-Левина-Полевого [19] (формула (А2) приложения, подробнее см. в [20]). Указанные кривые соответствуют вкладам второго и первого членов в квадратных скобках (А2) для электромагнитных мод с $S$-поляризацией и $P$-поляризацией. Следует отметить, что в (А2) учитываются как ближние (неоднородные), так и распространяющиеся (однородные) моды, хотя при малых значениях ширины щели $l$ однородные моды вносят малый вклад в общий результат. Кривая, показанная длинными штрихами на

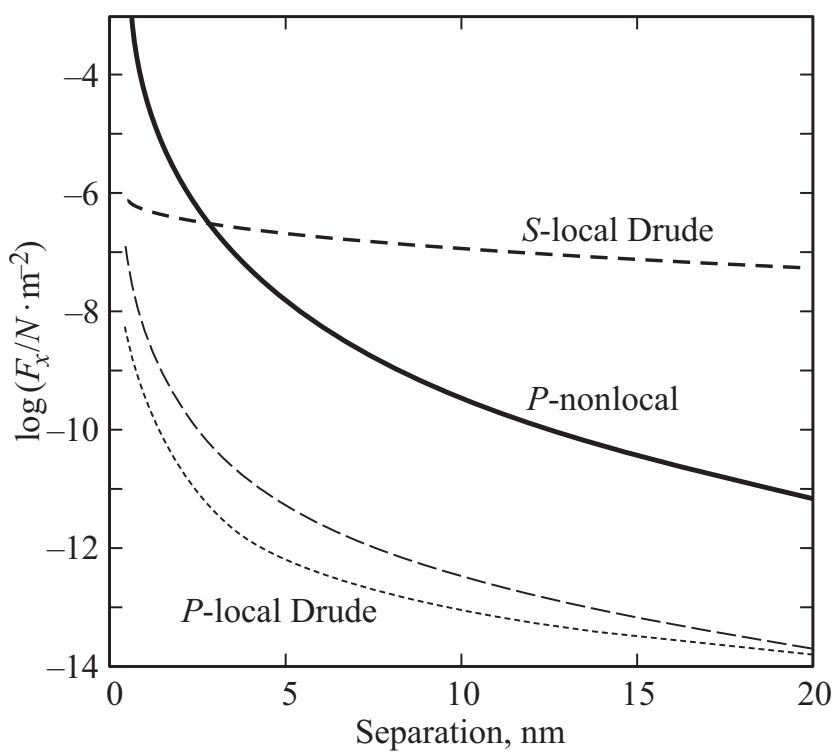

Рис. 4. Сила трения между двумя пластинами золота как функция расстояния $l$ при $T_{1}=T_{2}=300 \mathrm{~K}, V=1 \mathrm{~m} / \mathrm{s}$. Кривая $P$-nonlocal: формула (30); кривая $S$-local Drude: второе слагаемое (A2), определяющее вклад $S$-мод; кривая $P$-local Drude: первое слагаемое (А2), определяющее вклад $P$-мод; тонкая штриховая кривая: формула (35). Вклад $s$-поляризации в нашей нерелятивистской нелокальной теории $(c \rightarrow \infty)$ отсутствует.

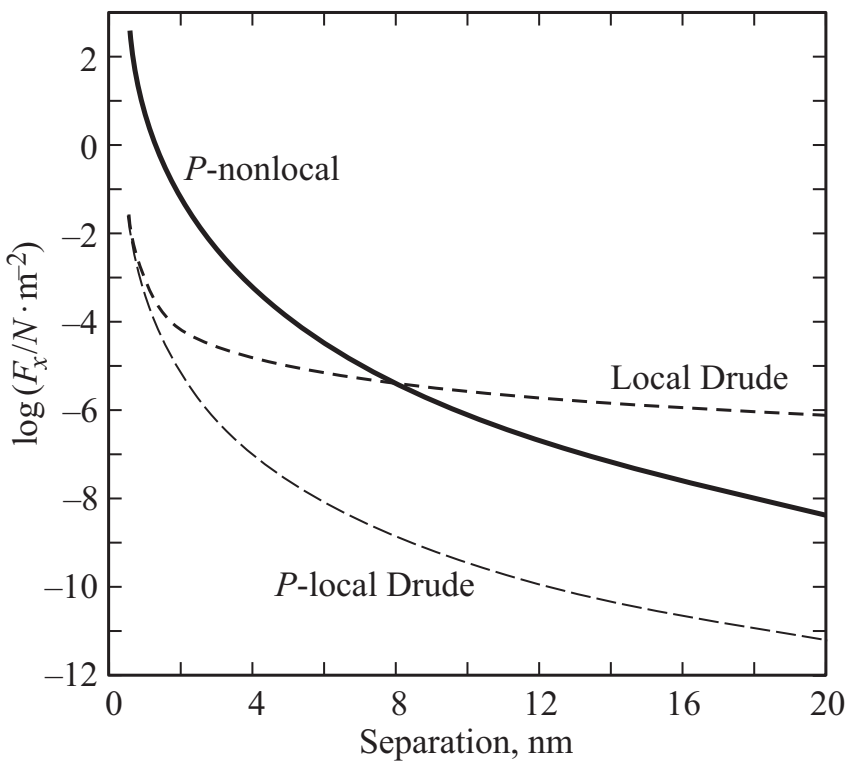

Рис. 5. Сила квантового трения двух пластин золота как функция расстояния $l$ при $V=0.1 V_{F}\left(V_{F}=1.4 \cdot 10^{6} \mathrm{~m} / \mathrm{s}\right.$. Толстая сплошная кривая (P-nonlocal): формула (31); кривая local Drude: формула (A3) (суммарный вклад $S$-мод и $P$-мод); тонкая штриховая линия ( $P$-local Drude) соответствует вкладу $P$-мод в (A3). Зависимость, соответствующая (35) (незапаздывающее приближение Друде для $P$-мод), близка к зависимости $P$-local Drude и поэтому не показана.

рис. 4, была рассчитана по формуле (34) и соответствует незапаздывающему локальному приближению Друде. Мы видим, что эффект ПД велик только при $l<2 \mathrm{~nm}$, тогда как при больших значениях $l$ доминирует вклад $S$-мод, рассчитанный в рамках локальной теории. Все кривые на рис. 4 удовлетворяют закону $F_{x} \sim V$, но зависимость от $l$ у них разная: $F_{x} \sim l^{-5}$ для $P$-nonlocal Drude, $F_{x} \sim l^{-0.9}$ для $S$-local Drude, и $F_{x} \sim l^{-4}$ для двух других кривых.

На рис. 5 проводится сравнение сил квантового трения при $T=0$ и $V=0.1 V_{F}$. Сплошная толстая кривая рассчитана по формуле (31). Штриховая толстая кривая рассчитана по формуле (А3) с учетом $P$-мод и $S$-мод. Кривая $P$-local Drude показывает вклад $P$-мод в (A3) (первое слагаемое в квадратных скобках). Значения сил, вычисленных по формуле (35) (т.е. вклад $P$-мод без запаздывания), очень близки к значениям сил на кривой $P$-local Drude (вклад $P$-мод с учетом запаздывания), поэтому на рисунке не показаны. Из рис. 5 следует, что диапазон расстояний $l$, при которых влияние ПД велико $(l<8 \mathrm{~nm})$ в этом случае шире, чем для сил трения при конечной температуре (рис. 4). Сплошная толстая кривая ( $P$-local Drude) соответствует зависимости $F_{x} \sim V^{3} / l^{7.2}$ при $1<l<2 \mathrm{~nm}$ и $F_{x} \sim V^{3} / l^{2}$ при $2<l<20 \mathrm{~nm}$. Кривая local Drude имеет зависимость $F_{x} \sim V^{3} / l^{3.2}$ при $1<l<2 \mathrm{~nm}$ и $F_{x} \sim V^{3} / l^{2}$ при $2<l<20 \mathrm{~nm}$. 
Мы также сравнили наши результаты с данными численного расчета [8], используя две реперные точки (из рис. $8, a, b[8])$, соответствующие скорости $V / V_{F}=0.2$ и ширине щели $1.27 \mathrm{~nm}\left(24 a_{0}\right)$ и $5.3 \mathrm{~nm}\left(100 a_{0}\right)$. Параметр модели желе $\left(r_{s}=3 a_{0}\right)$ в [8] близок к нашему $\left(r_{S}=3.01 a_{0}\right)$. Согласно данным [8], для указанных расстояний $F_{x} \approx 9 \mathrm{~N} / \mathrm{m}^{2}$ и $F_{x} \approx 0.004 \mathrm{n} / \mathrm{m}^{2}$, тогда как из(31) получаем $F_{x}=10.8 \mathrm{~N} / \mathrm{m}^{2}$ и $F_{x} \approx 0.0055 \mathrm{~N} / \mathrm{m}^{2}$, то есть имеется достаточно хорошее согласие. При этом заметим, что расчет силы $F_{x}$ в работе [8] является очень трудоемким, поскольку квантовая задача с вычислением волновых функций электронов системы и нахождением функции диэлектрического отклика пластин решается при каждом значении $l$, т. е. при заданной геометрии. Поэтому в [8] отсутствуют результаты расчета зависимости $F_{x}(l)$ в широком диапазоне значений $l$.

Несмотря на „положительное“ влияние ПД при малых расстояниях, приводящее к возрастанию сил трения, их абсолютные значения все же очень малы. Например, в идеальной конфигурации „сфера-плоскость“, используя приближение Дерягина $F_{s p} \approx \pi R a F_{x}(a, V)$ для взаимодействия сферы с плоскостью, где $R$ и $a$ - радиус сферического тела и его минимальное расстояние от плоскости, из (30) следует $F_{s p}=3 \cdot 10^{-15} N$ при $R=100 \mu \mathrm{m}, V=300 \mathrm{~m} / \mathrm{s}, a=1 \mathrm{~nm} T=300 \mathrm{~K}$. При этом $F_{x}=0.01 \mathrm{~N} / \mathrm{m}^{2}$. В свою очередь, в типичной экспериментальной ситуации, когда можно применить динамический метод АCM, даже использованное выше достаточно „умеренное“ значение скорости требует большой величины произведения амплитуды колебаний $A$ и частоты $f$, поскольку $2 \pi A f=300 \mathrm{~m} / \mathrm{s}$. Большие значения $A, R$ и малость $l$ затрудняют проведение такого эксперимента даже с имеющейся на сегодняшний день техникой позиционирования. Измерение квантовой силы трения выглядит еще сложнее, так как при $V=300 \mathrm{~m} / \mathrm{s}$ получается гораздо меньшее значение силы трения, $F_{x}=6 \cdot 10^{-8} \mathrm{~N} / \mathrm{m}^{2}$, или нужно увеличить скорость до значений порядка $10^{5} \mathrm{~m} / \mathrm{s}$. В этом случае, возможно, предпочтительнее исследовать скользящее взаимодействие с поверхностью ускоренных кластерных частиц (см. [3]).

Наконец, следует также обсудить роль температуры. В соответствии с (18), (30), нелокальная сила трения пропорциональна квадрату температуры и поэтому уменьшается при понижении температуры. Поскольку основным механизмом диссипации энергии при наличии ПД является возбуждение электрон-дырочных пар, эта зависимость вполне понятна. Что касается сил трения в локальном приближении Друде (с учетом $P$ - и $S$-мод), то в наших недавних расчетах [20] отмечалось резкое возрастание $F_{x}$ при $T<50 \mathrm{~K}$ при учете температурного изменения $\gamma$ - фактора по закону Блоха-Гюнайзена, (в выражении для диэлектрической проницаемости). Очевидно, вопрос о низкотемпературном изменении силы трения еще требует дополнительного исследования, поскольку в этом случае роль $\gamma$ - фактора может играть затухание Ландау, что отмечалось авторами [6].

\section{6. Заключение и выводы}

В статье изложен вывод выражений для сил трения ван-дер-Ваальса, действующих на частицу (толстую металлическую пластину), движущуюся с постоянной скоростью параллельно другой толстой металлической пластине (конфигурации 1 и 2). Теория является обобщением модели зеркального отражения и флуктуационно-электромагнитной теории с учетом пространственной дисперсии материала пластины. С использованием аналитического приближения для объемной диэлектрической проницаемости $\varepsilon(\omega, \mathbf{k})$ металла получены аналитические выражения для сил трения при конечной температуре $T$ и в случае квантового трения $(T=0)$. При обосновании выражений для сил трения в конфигурации параллельных пластин использован принцип соответствия между конфигурациями 1 и 2. Это обеспечивает однозначный предельный переход между формулами, полученными с локальной и нелокальной формой диэлектрической проницаемости.

Сравнение локального и нелокального приближений показывает, что силы трения ван-дер-Ваальса в рамках нелокальной теории на 2-4 порядка величины выше, чем в рамках локальной теории (как при конечной температуре, так и при $T=0)$. Формально математически это увеличение связано с большой величиной фактора $\omega_{p} / \gamma$, что согласуется с результатами $[8,9]$. Сравнение численных значений силы трения пластин со значениями, полученными в рамках квантово-полевой теории [8] также показывает хорошее согласие.

Основной теоретический результат статьи состоит в том, что развитая теория позволяет проводить расчеты сил трения ван-дер-Ваальса и квантового трения при расстояниях между телами $1-10 \mathrm{~nm}$ с учетом пространственной дисперсии диэлектрической проницаемости материалов.

\section{Приложение А}

В [20], преобразуя общее выражение для силы трения, полученное в теории Рытова-Левина-Полевого [19] с локальной формой диэлектрической проницаемости $\varepsilon(\omega)$, мы получили формулу для силы трения двух параллельных толстых пластин при их относительном движении с нерелятивистской скоростью $V / c \ll 1$ (величины, относящиеся к пластинам 1 и 2, снабжены индексами 1,2 )

$$
\begin{aligned}
F_{x}= & -\frac{\hbar}{4 \pi^{3}} \int_{0}^{\infty} d \omega \int d^{2} k k_{x}\left[\operatorname{Im}\left(\frac{q_{1}}{\varepsilon_{1}}\right) \operatorname{Im}\left(\frac{\tilde{q}_{2}}{\tilde{\varepsilon}_{2}}\right) \frac{|q|^{2}}{\left|Q_{e}\right|^{2}}\right. \\
& \left.+\operatorname{Im}\left(\frac{q_{1}}{\mu_{1}}\right) \operatorname{Im}\left(\frac{\tilde{q}_{2}}{\tilde{\mu}_{2}}\right) \frac{|q|^{2}}{\left|Q_{\mu}\right|^{2}}\right] \\
& \times\left[\operatorname{coth}\left(\hbar \omega_{-} / 2 T_{2}\right)-\operatorname{coth}\left(\hbar \omega / 2 T_{1}\right)\right] .
\end{aligned}
$$


Здесь $q=\sqrt{k^{2}-(\omega / c)^{2}}, q_{1}=\sqrt{k^{2}-\varepsilon_{1} \mu_{1}(\omega / c)^{2}}, q_{2}=$ $=\sqrt{k^{2}-\varepsilon_{2} \mu_{2}(\omega / c)^{2}}$, „тильда“ означает, что аргументы диэлектрической и магнитной проницаемости $\varepsilon_{1,2}$ и $\mu_{1,2}$ берутся при $\omega_{-}=\omega-k_{x} V$,

$$
\begin{aligned}
Q_{e} & =\left(q+q_{1} / \varepsilon_{1}\right)\left(q+\tilde{q}_{2} / \tilde{\varepsilon}_{2}\right) \exp (q l) \\
& -\left(q-q_{1} / \varepsilon_{1}\right)\left(q-\tilde{q}_{2} / \tilde{\varepsilon}_{2}\right) \exp (-q l),
\end{aligned}
$$

а величина $Q_{\mu}$ определяется таким же выражением с заменой $\varepsilon \rightarrow \mu$ Формула (A1) описывает силу трения, действующую на пластину 1 , движущуюся в направлении оси $x$ с постоянной скоростью $V$. Ее важными особенностями являются отсутствие в явной форме френелевских коэффициентов отражения и отсутствие разделения вкладов однородных и неоднородных мод на оси волновых векторов. Из (А1), в линейном приближении по скорости при $T_{1}=T_{2}=T$, следует (см. (23) в [20])

$$
\begin{aligned}
F_{x}= & \frac{\hbar V}{2 \pi^{2}} \int_{0}^{\infty} d \omega \frac{d n(\omega)}{d \omega} \int d^{2} k k_{x}\left[\operatorname{Im}\left(\frac{q_{1}}{\varepsilon_{1}}\right) \operatorname{Im}\left(\frac{q_{2}}{\varepsilon_{2}}\right) \frac{|q|^{2}}{\left|Q_{e}\right|^{2}}\right. \\
& \left.+\operatorname{Im}\left(\frac{q_{1}}{\mu_{1}}\right) \operatorname{Im}\left(\frac{q_{2}}{\mu_{2}}\right) \frac{|q|^{2}}{\left|Q_{\mu}\right|^{2}}\right],
\end{aligned}
$$

а при $T_{1}=T_{2}=0$, соответственно, - выражение для квантовой силы трения $[18,20]$

$$
\begin{aligned}
& F_{x}=\frac{\hbar}{4 \pi^{3}} \int_{-\infty}^{+\infty} d k_{y} \int_{0}^{\infty} d k_{x} k_{x} \\
& \times \int_{0}^{k_{x} V} \exp (-2 k l) \operatorname{Im} \Delta_{1 \varepsilon} \operatorname{Im} \tilde{\Delta}_{2 \varepsilon}\left|D_{\varepsilon}\right|^{-2}+(\varepsilon \rightarrow \mu) .
\end{aligned}
$$

Здесь $\quad \Delta_{i \varepsilon}=\left(\varepsilon_{i} q_{i}-q_{i}\right) /\left(\varepsilon_{i} q_{i}+q_{i}\right), \quad i=1,2, \quad D_{\varepsilon}=$ $=1-\Delta_{1 \varepsilon} \tilde{\Delta}_{2 \varepsilon} \exp (-2 k l)$, а для соответствующих магнитных вкладов нужно сделать замену $\varepsilon \rightarrow \mu$. Заметим, что в п. 3 расматривался случай немагнитных материалов, когда $\mu_{1,2}=1$.

\section{Конфликт интересов}

Авторы заявляют, что у них нет конфликта интересов.

\section{Список литературы}

[1] M. Aspelmeyer, T.J. Kippenberg, M. Marquardt. Rev. Mod. Phys. 86, 1391 (2014).

[2] K.A. Milton, J.S. Høye, I. Brevik. Symmetry 8, 29 (2016).

[3] Г.В. Дедков, А.А. Кясов. УФН 187, 599 (2017).

[4] A.I. Volokitin, B.N.J. Persson. Electromagnetic fluctuations at the nanoscale. Theory and applications. Springer-Verlag, Berlin, Heidelberg (2017).

[5] G.L. Klimchitskaya, V.M. Mostepanenko. Proc. Peter the Great St. Petersburg Polytech. Univ. 1, 41 (2015); Mod. Phys. Lett. A 34, 2040007 (2020).
[6] R. Esquivel, V.B. Svetovoy. Phys. Rev. A 69, 062102 (2004).

[7] V. Despoja, M. Sunjic, L. Marusic. Phys. Rev. B 75, 045422 (2007).

[8] V. Despoja, P.M. Echenique, M. Sunjic. Phys. Rev. B 83, 205424 (2011).

[9] D. Reiche, D.A.R. Dalvit, K. Busch, F. Intravaia. Phys. Rev. B 95, 155448 (2017).

[10] R.H. Ritchie, A.L. Marusak. Surf. Sci. 4, 234 (1966).

[11] R. Nunez, P.M. Echenique, R.H. Ritchie. J. Phys. C 13, 4229 (1980).

[12] F.J. Garcia de Abajo, P.M. Echenique. Phys. Rev. B 46, 2663 (1992).

[13] G.V. Dedkov, A.A. Kyasov. Surf. Sci. 604, 562 (2010).

[14] Г.В. Дедков, А.А. Кясов. ФТТ, 44, 1729 (2002).

[15] T.L. Ferrell, P.M. Echenique, R.H. Ritchie. Solid. State Commun. 32, 419 (1979).

[16] E.M. Lifshitz. Sov. Phys. JETP 2, 73 (1956).

[17] N.D. Mermin. Phys. Rev. B1, 2362 (1970).

[18] J.B. Pendry. J. Phys. Condens. Matter 9, 10301 (1997).

[19] V.G. Polevoy. Sov. Phys. JETP 71, 1119 (1990).

[20] Г.В. Дедков, А.А. Кясов. ФТТ 60, 2311 (2018). 